Communications in Physics, Vol. 19, No.1 (2009), pp. 1-6

\title{
A SUPERLUMINAL FORMALISM FOR MAJORANA-LIKE LEPTON
}

\author{
VO VAN THUAN \\ Vietnam-Auger Cosmic Ray Laboratory (VATLY) \\ Institute for Nuclear Science and Technology (INST) \\ 179 Hoang Quoc Viet Street, Nghia Do, Hanoi, Vietnam \\ *E-mail:vvthuan@vaec.gov.vn
}

\begin{abstract}
This work deals with the nature of Majorana particles by applying the timelike formalism of the superluminal Lorentz transformation (SLT). It is proposed that along with the SLT of the space-time coordinates, the Dirac equation should be treated simultaneously by a Majorana-like representation to be invariant. This formalism leads to a natural understanding of Majorana physics.
\end{abstract}

\section{INTRODUCTION}

The paper of Majorana published more than 70 years ago [1], at the beginning was applied for a symmetrical view on electron and positron. However, it was found later that the represented formalism is not for electron-positron, but describes neutral leptons, probably, a new kind of hypothetical neutrinos, which differ from Dirac neutrinos by the identical symmetry between particle and anti-particle. While massless Dirac and Majorana neutrinos seem to be indistinguishable and well described by the Standard Model (SM) where only their left-handed eigenstates can interact with the gauge fields, the neutrino oscillation implies that neutrino should have non-zero mass. Along with the traditional tendency of developing the SM to generate a normal mass and let the right-handed neutrinos show up, we proposed a model of space-time symmetry as an alternative approach which considers neutrino as time-like leptons, traveling in the flat $3 \mathrm{D}$-time while twisting in the 3D-space [2]. The first step in the present study is to formulate a formalism to understand the physics of massive superluminal leptons in the frame of which the superluminal leptons would formally satisfy Majorana physics.

\section{FORMALISM OF THE SUPERLUMINAL LORENTZ TRANSFORMATION (SLT)}

The $\{1,3\}$ Minkowski time-space (with geometrical unit $c=1$ ) corresponds to the flat pseudo-Euclid geometry as follows:

$$
d s^{2}=d t^{2}-d x^{2}-d y^{2}-d z^{2}
$$

Let's consider a material point moving in Minkowski time-space. The superluminal Lorentz transformation suggested by Recami [3] seems to keep a real 3D-Euclid space $\left(x^{\prime}, y^{\prime}, z^{\prime}\right)$, 
however, the two transverse coordinates are, indeed, imaginary in opposite to the real longitudinal space axis. Therefore, in application of the formal SLT we link these two transverse axes with the (longitudinal) time axis to form a 3D-Euclid time. Following Recami [3] we introduce the SLT from a subluminal reference frame $K$ to a superluminal reference frame $K^{\prime}$ as:

$$
\begin{aligned}
& z=\gamma\left(t^{\prime}-\beta z^{\prime}\right) ; \quad x=i . x^{\prime}=v^{\prime} \\
& t=\gamma\left(z^{\prime}-\beta t^{\prime}\right) ; \quad y=i . y^{\prime}=w^{\prime}
\end{aligned}
$$

Recami [3] suggested that for a material point (or a particle) moving faster than light $\left(\beta^{\prime}>1\right)$, there are two operations needed in the SLT. Firstly, it turns up the relative speed $\left(\beta=1 / \beta^{\prime}\right)$, which is equivalent to turn time axes to spatial ones and vice-versa; then $\gamma=\sqrt{1-\beta^{2}}$. Secondly, it converts all imaginary variables into real ones to meet the physical reality. At variance with [3] we propose to replace the imaginary "space" coordinates $\left(x^{\prime}, y^{\prime}\right)$ in $(2)$ by the real time-like coordinates $\left(v^{\prime}, w^{\prime}\right)$. Such a SLT converts a $\{1,3\}$-Minkowski time-space $\{t, x, y, z\}$ with the geometry (1) to a $\{3,1\}$ time-space $\left\{v^{\prime}, w^{\prime}, t^{\prime}, z^{\prime}\right\}$ with the following quadratic equation:

$$
d s^{2}=d z^{\prime 2}-d v^{\prime 2}-d w^{\prime 2}-d t^{\prime 2}
$$

For the tachyon in according to the transformation (2) for an economic version we may imply a dual role to the transverse time axes $\left(v^{\prime}, w^{\prime}\right)$, namely, the same axes play a role of transverse times for tachyon and simultaneously, a role of transverse real space $(x, y)$ for bradyon and for us, as subluminal observers. Consequently, the equation (3) can be rewritten as:

$$
d s^{2}=d x^{\prime 2}+d y^{\prime 2}+d z^{\prime 2}-d t^{\prime 2}=d z^{\prime 2}-d x^{2}-d y^{2}-d t^{\prime 2}
$$

The geometry in (3) and (4) is identical to the SLT in (2). The corresponding energymomentum relation of tachyon is:

$$
p_{z^{\prime}}^{2}-\vec{E}^{2}=m^{2}>0
$$

It is to emphasize that in (5) the momentum is single directional, while the energy is three dimensional in according to our definition of the superluminal space and time. The equation (5) may be rewritten conventionally with a formal 3D-momentum presentation as in [3]:

$$
E_{t^{\prime}}^{2}-p_{x^{\prime}}^{2}-p_{y^{\prime}}^{2}-p_{z^{\prime}}^{2}=E_{t^{\prime}}^{2}-\left(i . p_{x}\right)^{2}-\left(i . p_{y}\right)^{2}-p_{z^{\prime}}^{2}=\mu^{2}=(i . m)^{2}<0
$$

However, such formal 3D-momenta $p_{x}^{\prime}, p_{y}^{\prime}, p_{z}^{\prime}$ can not form a real 3D-Euclid momentum space, because the "transverse momenta" are imaginary.

\section{REPRESENTATION OF DIRAC EQUATION FOR ELECTRON-POSITRON}

Let's recall the traditional Dirac theory of free electron where the wave functions are complex and derive from a system of two equations, the primary Dirac equation and its conjugate:

$$
\gamma_{4} E \psi=i . \gamma_{k} p_{k} \psi+m \psi
$$




$$
-E \bar{\psi} \gamma_{4}=i \cdot p_{k} \bar{\psi} \gamma_{k}+m \bar{\psi}
$$

Originally, from the traditional Dirac electron-positron theory, each of the equations has a general solution, the four-component wave functions $\psi$ or $\bar{\psi}$ associated with both positive and negative time-energy sub-solutions, correspondingly. The time-energy dependence of a wave function is often expressed by a term of the form: $\psi \sim e^{-i \Omega . t}$ where $\Omega$ is de-Broglie frequency of electron. In the momentum representation, instead of changing the sign of energy and time, the later is kept positive but the frequency $\Omega$ adopts both positive and negative signs. Namely, $\psi=\psi_{+}+\psi_{-}$, where $\psi_{+}$is a function of positive frequency, while $\psi_{-}$is of the negative one. In fact, the reality seems to need only four eigenstates: two states of electron and positron, each of which has two sub-states of opposite spin projections, while the above equation system gives twice more solutions. For a reduction, it was assumed that only the positive energy is realistic, then instead of the sub-solution of the negative frequency, the second-conjugated equation in (7) is treated under C-operator which partly produces positron of positive energy, replacing the electron solution of negative energy. Such a combined operation is called reinterpretation principle (RIP). Consequently, the traditional Dirac formalism leads to a realistic solution as $\psi=\psi_{+}+\psi_{-} \Rightarrow\left(\psi_{+}^{e}+\psi_{+}^{p}\right)$; where $\psi_{-}$is replaced by $\psi_{+}^{p}=C \bar{\psi}_{-}$, a positron solution with positive energy. However, the RIP solution is not identical to the general solution of Dirac equation because $\psi$ and $\bar{\psi}$ are not solutions of the same equation.

In the present study, for an alternative discussion relating to the superluminal formalism, we propose to replace the Dirac's RIP by the time reversion as following: We assume, firstly, that electron has a time-like spin or t-spin equal $1 / 2$ of which the projection on the longitudinal time axis (t-helicity) should correlate strictly with the sign of the frequency $\Omega$ and then, with the electrical charge. Secondly, for a more natural consideration, we operate the time reversion $T$ which converts the time axis (and the sign of energy) and simultaneously changing the electrical sign to the opposite, i.e. equivalent to C-operator in RIP, then $T \psi_{-}=C \bar{\psi}_{-}$getting now an eigenstate of positron evolving toward the future together with electron. Indeed, if $\psi \sim e^{-i \Omega . t}$ is the unique form of time evolution, the complex conjugation $\bar{\psi}$ equivalent to a reflection of time $\psi(t) \leftrightarrow \psi(-t)$, as well as energy. Acting the T-operator on Dirac equation (7) in combining with matrix transposition should lead to the corresponding conjugate equation of the $\left(T \psi_{-}\right)$function as follows:

$$
T\left[\gamma_{4} E \psi_{-}=i . \gamma_{k} p_{k} \psi_{-}+m \psi_{-}\right] \rightarrow\left[-E\left(T \psi_{-}\right) \gamma_{4}=i . p_{k}\left(T \psi_{-}\right) \gamma_{k}+m\left(T \psi_{-}\right)\right]
$$

where $T=\gamma_{1} \gamma_{2} \gamma_{3}$. In this consideration we imply that the sign of electrical charge links with the projection of t-spin of electron. Indeed, during the T-operation while time $-t$ changes to $+t$, the projection of $\mathrm{t}$-spin as an axial vector should flip back relatively to $+t$. Instead of the solution $\psi_{-}$of equation for an electron with negative energy and evolving

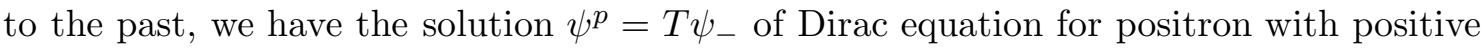
energy and evolving toward the future. We conclude that the Dirac equation and its conjugate for the positive solution describe the motion of electron; while the same pair of Dirac equations for the negative solution describe the motion of positron.

Each of the two solutions in Equations (7) corresponding to electron and positron, has only two linear independent subsolutions of two opposite spin's projections of electron or 
positron. Therefore, in case of a maximal mixing it is applied to electron and positron as follows:

$$
\begin{aligned}
\psi^{e} & =\frac{1}{\sqrt{2}}\left(\left|\psi_{+1 / 2}^{e}\right\rangle+\left|\psi_{-1 / 2}^{e}\right\rangle\right) \\
\psi^{p} & =\frac{1}{\sqrt{2}}\left(\left|\psi_{+1 / 2}^{p}\right\rangle+\left|\psi_{-1 / 2}^{p}\right\rangle\right)
\end{aligned}
$$

The combinations (8) describe the most natural stable states of lepton beam (electron or positron) in a dynamic equilibrium with the interacting medium.

\section{REPRESENTATION OF DIRAC EQUATION FOR SUPERLUMINAL LEPTON}

Based on the superluminal geometry (4) and equation (6) we write a "formal" Dirac equation for tachyon in the momentum representation as follows:

$$
\gamma_{4} E_{t^{\prime}} \psi^{\prime}=\left(-\gamma_{1} p_{x}-\gamma_{2} p_{y}+i \cdot \gamma_{3} p_{z^{\prime}}\right) \psi^{\prime}+\mu \psi^{\prime}
$$

This expression differs from the subluminal Dirac equation by the imaginary transverse "momenta" and the mass terms. Now applying a Majorana-like representation $\psi=U_{M} \cdot \psi^{\prime}$, where:

$$
U_{M}=\frac{1}{\sqrt{2}}\left(\gamma_{4}+\gamma_{3}\right)=\frac{1}{\sqrt{2}}\left(\begin{array}{cc}
I & -i . \sigma_{3} \\
i . \sigma_{3} & -I
\end{array}\right)
$$

we turn equation (9) into:

$$
\gamma_{3} E_{t^{\prime}} \psi=\left(\gamma_{1} p_{x}+\gamma_{2} p_{y}\right) \psi+i \cdot \gamma_{4} p_{z^{\prime}} \psi+\mu \psi
$$

Applying the version of the physical geometry (3), we turn back: $p_{x}=E_{v^{\prime}}=E_{1}, p_{y}=$ $E_{w^{\prime}}=E_{2}, E_{t^{\prime}}=-E_{3}$ and $p_{z^{\prime}}=p_{z}$, then rewrite (11) as:

$$
\gamma_{4} p_{z} \psi=i \cdot \gamma_{k} E_{k} \psi-m \psi
$$

We found that the Majorana-like equation (12) and its conjugate are exact as the form of Dirac equations(7) only with an exchange of the roles of energy and momentum which proves that instead of treating subluminal electron-positron the new equations govern superluminal leptons.

\section{MAJORANA PHYSICS IN THE SUPERLUMINAL FRAMES}

We are extending a similar analysis of the subluminal Dirac equations for electronpositron (7) now to solving the equation (12), for superluminal leptons. Similar to the action of T-operator at the subluminal frame on Dirac equation, here an action by Poperator (the space convertor) in combination with matrix transposition is applied on the solution with negative momentum, which coverts this solution into a right-handed one, but with positive momentum. Therefore, we can write the eigenstates of a free superluminal 
lepton as follows:

$$
\begin{aligned}
& \psi_{L}=\frac{1}{\sqrt{2}}\left(\left|\psi_{+}^{L}\right\rangle+\left|\psi_{-}^{L}\right\rangle\right) \\
& \psi_{R}=\frac{1}{\sqrt{2}}\left(\left|\psi_{+}^{R}\right\rangle+\left|\psi_{-}^{R}\right\rangle\right)
\end{aligned}
$$

in which $\psi_{L}$ is a wave function for the left-handed helicity and $\psi_{R}$ is another wave function for the right-handed helicity. They are two different superpositions of maximal mixing of two states $\psi_{+}$and $\psi_{-}$which are regarded, formally as the eigenstates of particle and antiparticle, respectively. For SLT formalism, superluminal leptons exist in a flat 3D-time being adopted as a realistic time-like 3D space, in which again we propose that a time-like spin (or simply t-spin, 1/2) of the superluminal lepton is able to rotate (in analogue to the s-spin of electron or positron orientable in $3 \mathrm{D}$ space). In case the maximal mixing (13) is keeping invariant, as the most stable states, $\psi_{L}$ and $\psi_{R}$ are the wave functions of superluminal Majorana particles, because they are identical in the relation between particle and anti-particle with well-conserved helicity.

In a complete similarity to (8), superpositions (13) can be also considered as the states of Majorana-like particle evolving to the future and back to the past, without Dirac's RIP. A half of Majorana-like leptons evolving to the past are almost sterile from subluminal observations.

Similar to electrons which may be polarized due to interaction with a polarizer and able to change their mixing in (8) between the two states $\psi_{+1 / 2}$ and $\psi_{-1 / 2}$ with opposite helicities, Majorana particles may also oscillate between eigenstates $\psi_{+}$and $\psi_{-}$and send a part of them to the past as sterile particles. For a total t-spin polarization we get a pure left-handed particle $\psi_{+}^{L}$ (or right-handed anti-particle $\psi_{-}^{R}$ ), which seems to be nothing else as a Dirac eigenstates with both labels: helicity and lepton charge. Therefore, it implies that the notions of Dirac neutrino and Majorana neutrino are relative, because they may oscillate to each other, depending on the mixing proportion in (13). However, such a definition of Dirac neutrino is not complete because the eigenstates in (13) are superluminal, while Dirac neutrinos, in their origin, should exist in 3D-space of the subluminal frames. As a result, we found that the superluminal leptons are identified by their helicity. Formally, we can assume in according to Parker [4] that instead of electric charge the superluminal lepton should have a magnetic monopole. We assume further that the sign of monopole should be well correlated with helicity of Majorana-like particle.

\section{CONCLUSION}

We found that along with the superluminal Lorentz transformation (SLT) of time and space coordinates, the quantum mechanical equations for leptons should be treated simultaneously by Majorana-like reinterpretation (10) to convert to an appropriate form of Dirac equation for superluminal lepton. The later is shown up as Majorana-like particle, which conserves strictly its helicity even small real masses can be produced, as neutrino oscillation implies recently.

The proposed formalism is not yet realistic, as there is an obvious asymmetry between electron and neutrino which demands the next step of the study to understand the nature 
of the mass of Majorana leptons and a suitable mechanism of violation of the spacetime symmetry. As expected, the proposed model would shed light on those mysterious particles and the origin of their P-nonconservation. This would also set new constraints on neutrinoless double beta decay.

\section{ACKNOWLEDGMENTS}

The author is indebted to Pierre Darriulat (VATLY, INST) and Nguyen Anh Ky (IOP) for useful discussions. The deep thanks is extended to VATLY members for their cooperation. The research has been funded by the National Basic Research Program for Physics of the Ministry of Science and Technology (MOST) of Vietnam.

\section{REFERENCES}

[1] E. Majorana, Nuovo Cim. 14(1937) 171.

[2] Vo Van Thuan, Proc. of the Osaka-Hanoi Forum 2005 on Frontiers of Basic Science, 2005, Hanoi, Vietnam, Sept. 27-29, 2005. Ed. H. Takabe et al., Osaka University Press. 2006, p.98.

[3] E. Recami and R. Mignani, Lett. Nuovo Cim. 4 (1972) 144.

[4] L. Parker, Phys. Rev. 188(1969) 2287.

Received 18 August 2008. 